\title{
Tactics Optimization of Percutaneous Coronary Intervention in the Area of Bifurcation of the Anterior Descending Artery By Means Of Cardiography from the Coronary Sinus
}

\author{
Shevchenko Yury Leonidovich ${ }^{1^{*}}$,Ermakov Dmitry Yuryevich ${ }^{1}$, Vahrameeva Anastasia Yuryevna ${ }^{1}$ \\ ${ }^{1}$ St.George Clinic of Thorasic and Cardiovascular Surgery, Pirogov National Medical and Surgical Center, the Russian Federation, Moscow. \\ *Corresponding Author: Shevchenko Yury Leonidovich, St. George Clinic of Thorasic and Cardiovascular Surgery, Pirogov National \\ Medical and Surgical Center, the Russian Federation, Moscow.
}

Received Date: July 13, 2020; Accepted Date: July 20, 2020; Published Date: August 11, 2020

Citation: Shevchenko Y Leonidovich, Ermakov D Yuryevich, Vahrameeva A Yuryevna, (2020) Orthostatic Hypotension in a Complex Clinical Setting Use of the Heart Rate Increase/Systolic Blood Pressure Decrease Ratio as a Diagnostic Aid. J Clinical Case Reports and Studies, 1(6): Doi: $10.31579 / 2690-8808 / 017$

Copyright: () 2020. Shevchenko Yury Leonidovich. This is an open-access article distributed under the terms of the Creative Commons Attribution License, which permits unrestricted use, distribution, and reproduction in any medium, provided the original author and source are credited.

\section{Abstract}

Introduction: The aim of this investigation is to evaluate the possibilities of the method of cardiography from the coronary sinus (ECG-CS) in optimization of stenting bifurcation lesions (BL) of coronary arteries (CA).

Materials and Methods: 43 patients with stable CHD with pseudo-BL of the anterior descending artery (ADA) were included in this investigation. We placed a 10-canal electrode into their coronary sinus at the beginning of percutaneous coronary intervention (PCI). Ischemic guidance was done with the WorkMate electrophysiological recording system.

Results: In 11 patients (25\%) out of 43 with CHD, the diagonal branch (DB) comprometation and ischemia dynamics ( $\mathrm{p}<0.05)$ in intracardiac lead (CS 3-4) after ADA stenting were discovered according to ECG-CS. Nine patients (20.9\%) underwent successful provisional-T-stenting, while a second stent was required for the other 2 patients.

Conclusion: ECG-CS provides the possibility to optimize coronary intervention in the area of CA bifurcation.

Keywords: Electrocardiography, ECG of the coronary sinus, Bifurcational lesion of the coronary arteries, Control of ischemia of myocardium.

\section{Introduction}

Bifurcation stenosis of the coronary arteries is referred to as a "complicated" lesion from the viewpoint of the interventional treatment of CHD, and accounts for $15-20 \%$ of the total number of atherosclerotic lesions of the coronary bed (CB) $[7,10]$. The difficulty in treating the condition is due to the multiple anatomic variants of bifurcation and hemodynamic changes during angioplasty, as these variants make it largely impossible to use the same strategy of interventional treatment for all patients with a bifurcation lesion of CA [7, 10, 12]. The most notable shortcoming of the existing methods of bifurcation stenting is an extensive local "metallization" of a stented artery due to applying one stent to another, in addition to the disturbance of the safety of the polymerdrug layer of stents during operations. These current flaws are often the reasons for restenosis developing and that of arterial bifurcation thrombosis as well, reducing the clinical efficiency of bifurcation stenting in the future [12]. At present, the main strategy of endovascular treatment for most patients with BL is based on stenting in the main vessel (MV) of bifurcation. In the case of optimal results, the dilatation of both branches with the help of kissing angioplasty (provisional T-stenting) is typically used, whereas double stenting methods are used mainly in patients with unsatisfactory clinical angiographical results of the provisional T-stenting (D, F-type dissection, considerable angious pain with negative dynamics of the ECG, bloodstream < TIMI III on the side branch (SB) [7, 10, 12].

Thus, the main problem while choosing the optimal amount of intervention related to $\mathrm{BL}$ in most cases is obtaining an objective and exact evaluation of the degree of comprometation of SB ostium after stenting in MV [10]. BL angiography is complicated to perform and interpret due to a number of reasons, such as the complicated geometry of $\mathrm{CA}$, presence of other side branches, intercrossing of vessels, and eccentric growth of atherosclerotic plaque in SB. Nowadays during bifurcational intervention related to $\mathrm{CA}$, to evaluate the functional importance of stenosis of SB, doctors use a method of invasive tests based on the concept of verifying ischemia via analysis of hemodynamic characteristics of intracoronary blood flow in several separate areas of the coronary bed: fractional flow reserve (FFR) and instantaneous wave-free ratio (iFR) $[10,11]$. 


\section{Fractional flow reserve}

The method of FFR has some limiting factors in order to be used in clinical practice. Therefore, one FFR measuring elongates the total time of intervention ( 7 minutes), the efficient dose of radiation $(2.8 \mathrm{mSv})$, and the volume of the introduced radio-opaque contrast agent $(35 \mathrm{~mL})$ [17]. Nevertheless, the main limit of using FFR is the necessity of a parenteral injection of vasodilator, as this is related to the risk of developing different side effects such as a sense of heat in the thoracic area, and the induction of life-threatening ventricular disturbances of heart rate [17]. Due to these factors, we do not do FFR for some patients. Thus, in 2017, according to the data given by the American College of Cardiology, doctors in the U.S. evaluated FFR during coronary intervention only in $6.1 \%$ of cases [16].

\section{Instantaneous wave-free ratio}

The method of detecting iFR cannot be completely validated with the aim of using it as a separate method of detecting myocardial ischemia (MyI) due to the characteristics of coronary blood flow in the area of CA stenosis. In 2020, we still do not have randomized trials where they compare the results of PCI on the basis of functionally evaluating stenosis through iFR to determine intervention tactics using angiographical data and optimal therapy. Likewise, there are very few investigations dealing with the hybrid protocol $\mathrm{FFR} / \mathrm{iFR}$, so it is difficult to prove this protocol's benefits, and the procedures of finding $\mathrm{MyI}$ are complicated and elongated, which is why FFR/iFR is rarely used in practice in the catheterization laboratory [11]. Adding to the difficulty, methods of determining iFR and FFR imply complicated and elongated manipulations, and special instruments should be used for $\mathrm{CB}$ at different stages of intervention $[13,15]$.

\section{Electrocardiography from the coronary sinus}

A prospective alternative invasive test for optimizing intervention tactics in the area of CA bifurcation is the use of a new electrophysiological method of finding MyI - electrocardiography from the coronary sinus. This method was elaborated, suggested and used by Professor Yu.L. Shevchenko in 2019 at Pirogov National Medical and Surgical Center (The Russian Federation, Moscow) [15]. The exactness of ECG-CS relative to finding ischemia of myocardium during surgical intervention dealing with the major coronary artery has been described by Yu.L. Shevchenko et al. over the course of 2019 and 2020 in some of his articles $[13,14,15]$. According to ROC-analysis done by Yu.L. Shevchenko et al. in 2020, the sensibility of the ECG-CS method in the case of depression arising in ST-segment was $79.5 \%$, with $100 \%$ specificity. In the case of registering ST-segment elevation on the intracardiac cardiogram, the sensibility of the method was $83 \%$, with $100 \%$ specificity [13].

\section{Fundamental foundation of the possibility of using the ECG method from the coronary sinus to find ischemia of myocardium}

From the viewpoint of pathological physiology, coronary stenting of the main vessel with the successive comprometation of the SB ostium is an equivalent of stress for myocardium, due to the fact that cardiomyocytes are not yet ready for acute ischemia. Shifting the ion gradients of cells in the area of hypoperfusion determines the change of the heart's electric field analyzed by means of an electrophysiologic recording station [2, 4, 8]. According to theoretical and experimental models of the cardioelectric field, the formation of systolic and diastolic flows injuring the myocardium in the case of ischemia can have four possible types of distribution of potential: the rest potential (RP) in the area with ischemia is less (according to module) when compared to the areas with the normal perfusion - hypopolarization; the rest potential in the injured area is higher (according to module) when compared to RP in the area with the normal perfusion - hyperpolarization; action potential (AP) of the pathological area is less when compared to AP of the healthy area- hypodepolarization; and AP of the injured area is higher when compared to AP of the healthy area - hyperdepolarization. In this situation synergism of the first and third types of potential shifting is possible [2, $3,4,5,8]$. Placing an endocardial electrode (EE) into the CS provides the possibility to discover potential differences in the couples of cathodeanode electrodes at the moment of reducing coronary perfusion which is found in intracardiac leads of ECG, such as ST-segment shifting relative to an isoline. The amplitude of deviation is correlated with the degree of ischemia, and the deviation's direction (depression, elevation) is correlated with the localization of the ischemic center and the charge of the injured area [13]. Thus, we have the aim to evaluate the possibilities of the method of electrocardiography from the coronary sinus to optimize tactics of stenting CA bifurcation lesions.

\section{Materials and methods.}

\section{Study population.}

43 patients $(100 \%)$ who have stable CHD with a pseudo-bifurcation lesion of ADA and without hemodynamically important stenosis of the diagonal branches (DB) have been included in this investigation. For these 43 patients we performed PCI according to the plan between 20182020 at Pirogov National Medical and Surgical Center. All the patients signed an informed consent to take part in the trial. The independence ethics committee of Pirogov National Medical and Surgical Center approved the authors' desire to hold a trial. The criteria to exclude patients from the experiment were the following: unstable stenocardia, disturbance of heart rate, mitral insufficiency, degree III-IV, disturbance of renal function (creatine $>200 \mathrm{mcmol} / \mathrm{L}$ ), contraindication to have standard antiplatelet therapy, coronary artery bypass grafting in anamnesis, and an occluding lesion of the CA.

\section{Standard functional intraoperation follow-up of a patient's condition}

Doctors performed PCI according to the standard method on the background of a constant follow-up of ECG in three standard leads and in three augmented leads of the extremities of a patient, along with pulse oximetry and the indirect measuring of blood pressure on a 5-10 minute interval with the help of the multiple-parameter configured DatexOhmeda Cardiocap 5 monitor (GE).

\section{ECG from the coronary sinus.}

The WorkMate (St. Jude Medical) electrophysiological recording system was used in our trial for carrying out invasive intraoperation screening of myocardial ischemia. The catheterization of the CS was done via the subclavical vein by placing a 7F introducer. Under the guidance of X-ray an endocardial 10-canal electrode CS was placed into the CS in the left anterior oblique projection. In order to obtain exact diagnostic results related to myocardial ischemia, we used the bipolar method of the system with the following set up: joining of electrodes into five cathode-anode couples with the frequency range $30-500 \mathrm{~Hz}$, a $1 \mathrm{~cm}$ cut-off signal, and amplitude $0.3 \mathrm{mV} / \mathrm{cm}$. The digital analysis of the obtained data was carried out with the EP WorkMate Recording System computer program, version 4.3.2.

\section{Algorithm of performing percutaneous coronary intervention.}

During the first stage of intervention, the type of coronary bed lesion (the large diameter of the lateral branches in the area of bifurcation, absence of considerable stenosis of the DB ostium from the viewpoint of hemodynamics before the intervention, the low risk and shifting of atheromatous masses) and constant follow-up on the myocardial ischemia through ECG-CS made it possible to perform stenting of the main branch with the protection of a DB coronary guide wire without additional manipulations for all the patients. Unless angiography discovered 
considerable DB stenosis, we performed bifurcation coronary intervention using the method of provisional $\mathrm{T}$-stenting with percutaneous balloon angioplasty (PBA) of DB ostium by means of kissing-dilatation with two balloon catheters. In certain cases, such as if the kissing-dilatation was inefficient, a considerable residual stenosis of the DB ostium was preserved, the blood flow of the side branch was lower than TIMI III, SB dissection type D, F was developing, or there were negative dynamics of ECG-CS, we performed a total bifurcation Tstenting. The stent was placed by means of the coronary guide wire in the diagonal branch, starting from the ostium. The optimization of the stented area was done by means of kissing-dilatation, using two balloon catheters. After PCI the control angiography was performed in at least two orthogonal projections, and then analysis of ischemic dynamics was done. If the ECG-CS results are satisfactory, the instruments are usually removed.

\section{Statistics.}

We calculated statistical data using PC MSI bymeans of Microsoft Excel, and carried out the statistical analysis of data using Statistica 10 for Windows (StatSoftInc., USA). Our aim was to obtain tables, diagrams, graphs and analytical values: the structure (p) of average value $(\mathrm{M})$ and the standard deviation ( \pm sd). Quantitative variables have been described by the following: the number of patients, average arithmetical value (M), a standard deviation from the average arithmetical value, the 25 -th and 75-th percentiles, and the median. The difference is considered statistically reliable if the mistake is $p<0.05$. If $p$ is less than or equal to the required level, it means that the result is statistically reliable. The data is shown in the diagrams below.

\section{Results.}

We have made a CHD diagnosis for all 43 patients (100\%) as a result of clinical investigation, taking into consideration complaint analysis, anamnesis of disease, and objective methods of investigation. Clinical and angiographic data of patients are presented in Tables $\mathbf{1}$ and $\mathbf{2}$.

\begin{tabular}{|c|c|c|}
\hline \multicolumn{2}{|l|}{ Criteria } & Patients num ber \\
\hline \multicolumn{2}{|c|}{ Number of patients } & 43 \\
\hline \multirow{2}{*}{\multicolumn{2}{|c|}{ Males }} & $31(72.1 \%)$ \\
\hline & & $12(27.9 \%)$ \\
\hline \multicolumn{2}{|l|}{ Age } & $56.3 \pm 4.2$ \\
\hline \multirow[t]{4}{*}{$\mathrm{FC}(\mathrm{NYHA})$} & $\mathrm{I}$ & 0 \\
\hline & II & $16(37.2 \%)$ \\
\hline & III & $27(62.8 \%)$ \\
\hline & IV & 0 \\
\hline \multicolumn{2}{|c|}{ Hypertension } & $28(65.1 \%)$ \\
\hline \multicolumn{2}{|c|}{ Diabetes mellitus } & $4(9.3 \%)$ \\
\hline \multicolumn{2}{|c|}{ Hyperlipidem ia } & $30(69.8 \%)$ \\
\hline \multicolumn{2}{|l|}{ Obesity } & $21(48.8 \%)$ \\
\hline \multicolumn{2}{|l|}{ Smoking } & $19(44.2 \%)$ \\
\hline
\end{tabular}

Table 1. Pre-operation characteristics of patients according to anamnesis data

All the diagonal branches in the BL area are important from the standpoint of hemodynamics: the diameter of the DB ostium is $2.46 \pm 0.23 \mathrm{~mm}$. DB stenosis degree is $22 \pm 19.3 \%$ (Table 2).

\begin{tabular}{|l|c|}
\hline \multicolumn{1}{|c|}{ Values } & (n=43) \\
\hline ADA stenosis degree, \% & $79 \pm 8.1$ \\
\hline ADA lesion length, mm & $9.2 \pm 2.4$ \\
\hline Reference ADA diameter, mm & $3.0 \pm 0.4$ \\
\hline SYNTAX Score I & $8.07 \pm 1.94$ \\
\hline Medina classification & $26(60.4 \%)$ \\
\hline 1.1 .0 & $6(14 \%)$ \\
\hline 1.0 .0 & $11(25.6 \%)$ \\
\hline 0.1 .0 & $22 \pm 19.3$ \\
\hline Diameter stenosis of DB ostium, \% & $2.46 \pm 0.23$ \\
\hline Diameter of DB ostium, mm & \\
\hline
\end{tabular}

Table 2. Angiographic characteristics of patients 
The proper criteria of ischemia, determined through ECG-CS, are the following: ST-segment changes are higher than $0.1 \mathrm{mV}$. After the electrode was placed into the CS and until instruments were introduced into the coronary bed, we did not observe any ischemic changes $(p=N S)$. In Table 3, the dynamics of ST-segment during intervention into the ADA in the area of bifurcation are shown. During balloon inflation and ADA stenting using an endocardial electrode, the ischemic dynamics $(\mathrm{p}<0.05)$ in intracardiac CS 1-2 and CS 3-4 leads were registered. Superficial ECG changes $(\mathrm{p}<0.05)$ are shown in I lead (Table 3).

\begin{tabular}{|c|c|c|c|c|c|c|c|c|}
\hline \multirow[b]{3}{*}{$(\mathrm{mV})$} & \multirow{2}{*}{\multicolumn{2}{|c|}{ State of rest $(n=43)$}} & \multirow{2}{*}{\multicolumn{2}{|c|}{ Balloon inflation $(n=25)$}} & \multicolumn{4}{|c|}{ Stent placing } \\
\hline & & & & & \multicolumn{2}{|c|}{$\begin{array}{l}\text { Patients with balloon inflation } \\
\qquad(\mathrm{n}=25)\end{array}$} & \multicolumn{2}{|c|}{$\begin{array}{l}\text { Patients without balloon } \\
\text { inflation }(n=18)\end{array}$} \\
\hline & depression & elevation & depression & elevation & depression & elevation & depression & elevation \\
\hline \multicolumn{9}{|c|}{ Superficial ECG } \\
\hline 1 & $0.04 \pm 0.01$ & $0.03 \pm 0.01$ & $0.07 \pm 0.02^{*}$ & $0.06 \pm 0.03^{*}$ & $0.09 \pm 0.02 *$ & $0.1 \pm 0.02 *$ & $0.1 \pm 0.02^{*}$ & $0.1 \pm 0.03^{*}$ \\
\hline II & $0.03 \pm 0.01$ & $0.03 \pm 0.01$ & $0.04 \pm 0.01$ & $0.06 \pm 0.02$ & $0.07 \pm 0.02$ & $0.06 \pm 0.03$ & $0.08 \pm 0.01$ & $0.06 \pm 0.03$ \\
\hline III & $0.03 \pm 0.02$ & $0.04 \pm 0.01$ & $0.03 \pm 0.01$ & $0.03 \pm 0.01$ & $0.04 \pm 0.02$ & $0.04 \pm 0.01$ & $0.03 \pm 0.01$ & $0.05 \pm 0.01$ \\
\hline aVR & $0.02 \pm 0.01$ & $0.03 \pm 0.01$ & $0.02 \pm 0.01$ & $0.03 \pm 0.01$ & & $0.05 \pm 0.01$ & & $0.03 \pm 0.01$ \\
\hline $\mathrm{aVL}$ & $0.04 \pm 0,02$ & $0.03 \pm 0.01$ & $0.06 \pm 0.01$ & $0.04 \pm 0.01$ & $0.07 \pm 0.01$ & $0.06 \pm 0.03$ & $0.07 \pm 0.01$ & $0.07 \pm 0.02$ \\
\hline aVF & $0.02 \pm 0.01$ & $0.02 \pm 0.01$ & $0.03 \pm 0.02$ & $0.03 \pm 0.01$ & $0.05 \pm 0.02$ & & $0.06 \pm 0.01$ & \\
\hline \multicolumn{9}{|c|}{ Intracardial ECG } \\
\hline $\mathrm{CS}_{1-2}$ & $0 / 05 \pm 0.01$ & $0.03 \pm 0.02$ & $0.11 \pm 0.02^{*}$ & $0.12 \pm 0.04^{*}$ & $0.11 \pm 0.01^{*}$ & $0.14 \pm 0.03^{*}$ & $0.11 \pm 0.02^{*}$ & $0.15 \pm 0.03^{*}$ \\
\hline $\mathrm{CS}_{3-4}$ & $0.03 \pm 0.02$ & $0.04 \pm 0.01$ & $0.12 \pm 0.02^{*}$ & $0.11 \pm 0.03^{*}$ & $0.11 \pm 0.02^{*}$ & $0.12 \pm 0.02^{*}$ & $0.12 \pm 0.02^{*}$ & $0.12 \pm 0.02^{*}$ \\
\hline $\mathrm{CS}_{5-6}$ & $0.04 \pm 0.02$ & $0.03 \pm 0.01$ & $0.04 \pm 0.02$ & $0.07 \pm 0.01$ & $0.05 \pm 0.02$ & $0.07 \pm 0.03$ & $0.06 \pm 0.02$ & $0.04 \pm 0.02$ \\
\hline $\mathrm{CS}_{7-8}$ & $0.03 \pm 0.01$ & $0.05 \pm 0.01$ & $0.04 \pm 0.02$ & $0.05 \pm 0.02$ & $0.04 \pm 0.02$ & $0.03 \pm 0.02$ & $0.05 \pm 0.02$ & $0.05 \pm 0.03$ \\
\hline $\mathrm{CS}_{9-10}$ & $0.03 \pm 0.01$ & $0.04 \pm 0.02$ & $0.05 \pm 0.01$ & $0.03 \pm 0.01$ & $0.04 \pm 0.02$ & $0.05 \pm 0.03$ & $0.04 \pm 0.04$ & $0.05 \pm 0.04$ \\
\hline
\end{tabular}

$*-p<0,05$

Table 3. Ischemic dynamics of ST-segment during intervention into ADA in the area of bifurcation

After ADA stenting for 32 patients, although there had been angiographic signs of exacerbating stenosis of the DB ostium, ischemic changes were not registered in the superficial ECG and ECG-CS. Thus, we decided to stop PCI. In 11 patients after ADA stenting ischemic dynamics $(\mathrm{p}<0.05)$ in CS 3-4 lead were registered (Table 4).

\begin{tabular}{|c|c|c|c|c|}
\hline \multirow{2}{*}{} & \multicolumn{4}{|c|}{ After ADA stenting } \\
\cline { 2 - 5 } & \multicolumn{5}{|c|}{ (n= 32) } & \multicolumn{2}{c|}{ ( $\mathbf{n = 1 1 )}$} \\
\hline (mV) & depression & elevation & depression & elevation \\
\hline \multicolumn{5}{|c|}{ Superficial ECG } \\
\hline I & $0.04 \pm 0.01$ & $0.05 \pm 0,01$ & $0.05 \pm 0.01$ & $0.05 \pm 0.03$ \\
\hline II & $0.04 \pm 0.02$ & $0.04 \pm 0,02$ & $0.04 \pm 0.02$ & $0.04 \pm 0.02$ \\
\hline III & $0.04 \pm 0.03$ & $0.05 \pm 0,03$ & $0.03 \pm 0.01$ & $0.04 \pm 0.01$ \\
\hline aVR & & $0.03 \pm 0,04$ & & $0.03 \pm 0.01$ \\
\hline aVL & $0.03 \pm 0.02$ & $0.03 \pm 0,02$ & $0.05 \pm 0.02$ & $0.04 \pm 0.01$ \\
\hline aVF & $0.03 \pm 0.01$ & \multicolumn{5}{|c|}{ Intracardial ECG } \\
\hline \multicolumn{5}{|c|}{} \\
\hline CS $_{1-2}$ & $0.03 \pm 0.01$ & $0.04 \pm 0.02$ & $0.06 \pm 0.02$ & $0.07 \pm 0.01$ \\
\hline CS $_{3-4}$ & $0.03 \pm 0.01$ & $0.03 \pm 0.01$ & $0.1 \pm 0.01 *$ & $0.12 \pm 0.01 *$ \\
\hline CS $_{5-6}$ & $0.04 \pm 0.02$ & $0.04 \pm 0.01$ & $0.06 \pm 0.02$ & $0.07 \pm 0.02$ \\
\hline CS $_{7-8}$ & $0.02 \pm 0.01$ & $0.03 \pm 0.01$ & $0.03 \pm 0.01$ & $0.03 \pm 0.01$ \\
\hline CS $_{9-10}$ & $0.02 \pm 0.01$ & $0.04 \pm 0.02$ & $0.03 \pm 0.01$ & $0.04 \pm 0.02$ \\
\hline
\end{tabular}

$$
*-p<0.05
$$

Table 4. ST-segment dynamics after stenting of ADA in the area of bifurcation

The minimal depression in CS 3-4 lead was $0.09 \mathrm{mV}$, the maximal depression $0.11 \mathrm{mV}$, and elevation $0.1 \mathrm{mV}$ and $0.15 \mathrm{mV}$, respectively (A). In 32 patients we did not find ischemic changes to the ST-segment (B).
The maximal depression, found in CS 3-4 lead, was $0.06 \mathrm{mV}$, and the maximal elevation was $0.06 \mathrm{mV}$ (Figures 1 and 2). 

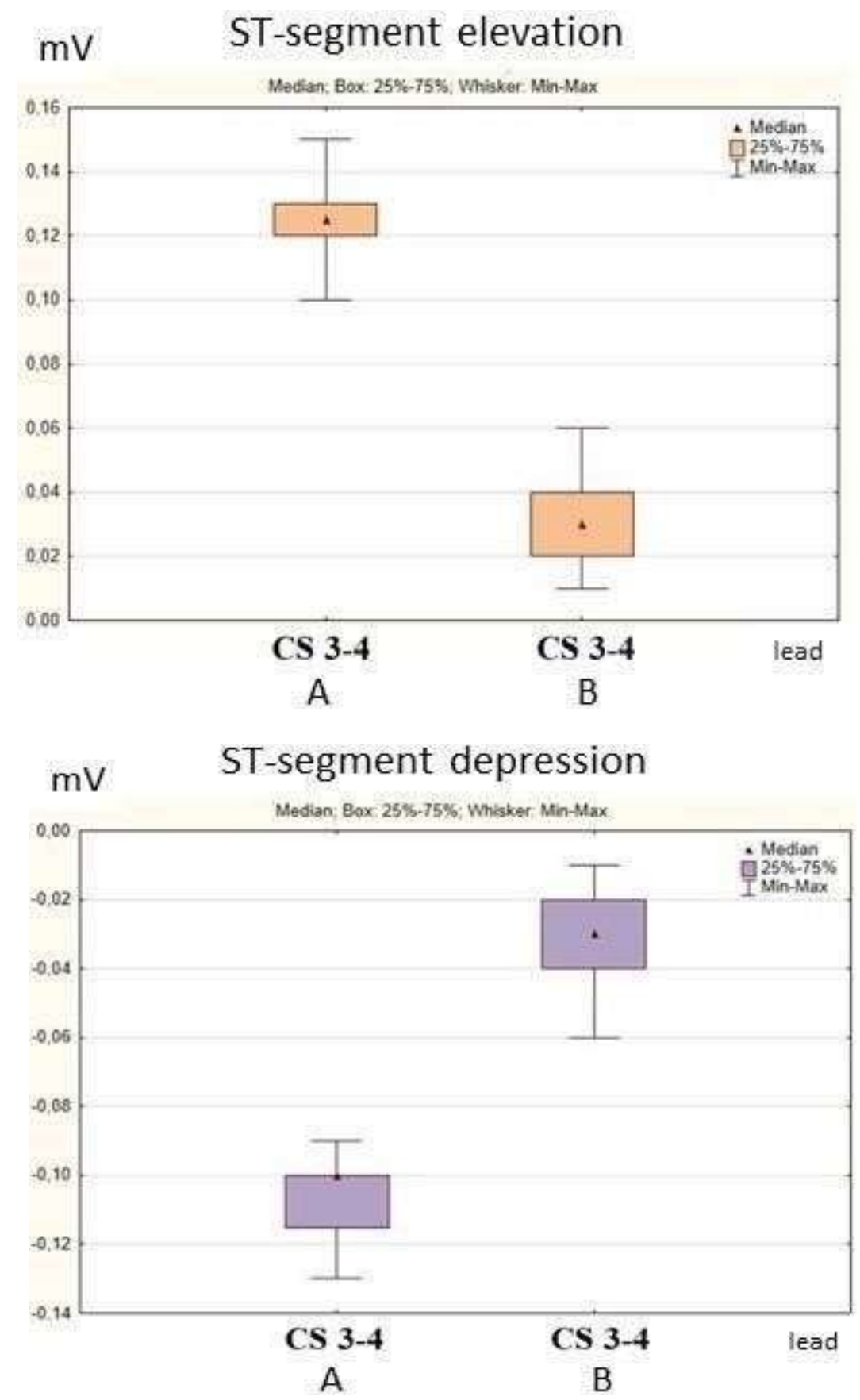

Figure 1. Dynamics of ST segment ECG-CS after ADA stenting, (A - SB comprometation, B - absence ofischemic changes).

Taking several things into consideration, such as the clinical presentation, the angiographic data of worsening DB stenosis, and theischemic changes in the intracardiac ECG, we decided to perform T-stenting of the DB ostium with PBA by means of the kissing-dilatation method (Table 5). 


\begin{tabular}{|c|c|c|c|c|c|c|}
\hline \multirow[b]{3}{*}{$(\mathrm{mV})$} & \multicolumn{4}{|c|}{ Intervention in the area of DB } & \multirow{2}{*}{\multicolumn{2}{|c|}{$\begin{array}{l}\text { At the end of intervention } \\
\qquad(n=11)\end{array}$}} \\
\hline & \multicolumn{2}{|c|}{$\begin{array}{l}\text { Patients with balloon inflation } \\
\qquad(\mathrm{n}=6)\end{array}$} & \multicolumn{2}{|c|}{$\begin{array}{c}\text { Patients without balloon } \\
\text { inflation }(n=5)\end{array}$} & & \\
\hline & depression & elevation & depression & elevation & depression & elevation \\
\hline \multicolumn{7}{|c|}{ Superficial ECG } \\
\hline $\mathbf{I}$ & $0.1 \pm 0.02^{*}$ & $0.1 \pm 0.01 *$ & $0.11 \pm 0.02^{*}$ & $0.11 \pm 0.01 *$ & $0.03 \pm 0.01$ & $0.04 \pm 0.01$ \\
\hline II & $0.05 \pm 0.01$ & $0.03 \pm 0.01$ & $0.02 \pm 0.01$ & $0.03 \pm 0.03$ & $0.03 \pm 0.02$ & $0.03 \pm 0.02$ \\
\hline III & & $0.06 \pm 0.02$ & & $0.05 \pm 0.03$ & $0.03 \pm 0.01$ & $0.04 \pm 0.02$ \\
\hline aVR & & $0.04 \pm 0.01$ & & $0.04 \pm 0.02$ & $0.06 \pm 0.01$ & $0.02 \pm 0.01$ \\
\hline $\mathrm{aVL}$ & $0.05 \pm 0.02$ & $0.05 \pm 0.01$ & $0.04 \pm 0.02$ & $0.05 \pm 0.02$ & $0.02 \pm 0.02$ & $0.02 \pm 0.02$ \\
\hline $\mathrm{aVF}$ & $0.03 \pm 0.01$ & $0.02 \pm 0.01$ & $0.05 \pm 0.01$ & $0.03 \pm 0.01$ & $0.02 \pm 0.011$ & \\
\hline \multicolumn{7}{|c|}{ Intracardiac ECG } \\
\hline $\mathrm{CS}_{1-2}$ & $0.1 \pm 0.03^{*}$ & $0.11 \pm 0.02^{*}$ & $0.11 \pm 0.03^{*}$ & $0.11 \pm 0.03^{*}$ & $0.02 \pm 0.01$ & $0.03 \pm 0.01$ \\
\hline $\mathrm{CS}_{3-4}$ & $0.11 \pm 0.01^{*}$ & $0.11 \pm 0.02^{*}$ & $0.12 \pm 0.02^{*}$ & $0.11 \pm 0.01^{*}$ & $0.04 \pm 0.01$ & $0.03 \pm 0.01$ \\
\hline $\mathrm{CS}_{5-6}$ & $0.05 \pm 0.03$ & $0.07 \pm 0.02$ & $0.05 \pm 0.01$ & $0.06 \pm 0.03$ & $0.04 \pm 0.01$ & $0.05 \pm 0.01$ \\
\hline $\mathrm{CS}_{7-8}$ & $0.05 \pm 0.01$ & $0.04 \pm 0.02$ & $0.04 \pm 0.01$ & $0.03 \pm 0.03$ & $0.04 \pm 0.01$ & $0.03 \pm 0.01$ \\
\hline$C_{9-10}$ & $0.04 \pm 0.02$ & $0.05 \pm 0.02$ & $0.03 \pm 0.02$ & $0.02 \pm 0.01$ & $0.02 \pm 0.01$ & $0.02 \pm 0.02$ \\
\hline
\end{tabular}

$*-\mathrm{p}<0,05$

Table 5. ST-segment dynamics during intervention in the area of DB ostium

After PBA of the DB ostium, done by the standard method and according to intracardiac ECG, ischemic changes were not registered for 9 patients $(\mathrm{p}=\mathrm{NS})$. In 2 patients, despite angioplasty of the LB ostium, angiographic signs of residual stenosis of the SB and depression in CS 3-4 led up to $0.12 \mathrm{mV}$ and the patients experienced pain in the retrosternal area. We thus decided to perform the total T-stenting of the DB. After the operation, the dynamics of the ST-segment were not registered in 11 patients.

After coronary stenting, none of the patients complained about stenocardia; when hemostasis occurred, we transferred all the study's patients into the intensive care ward for a day to allow for dynamic follow-up. During their time in intensive care, every patient's condition was evaluated as stable, in regards to functional (the standard dynamic ECG in 12 leads) and clinical criteria. Angiography was not required. After one day, each patient was transferred back to our department.

\section{Discussion.}

The most pertinent problem of PCI in the area of bifurcation when using the method of stenting with a double-guide-wire technique and provisional T-stenting is evaluating the functional importance of the SB for myocardium. The hemodynamic importance of ostium stenosis, unaccompanied by either reliable ischemic changes according to a standard ECG or if blood flow is lower than TIMI III, has not yet been determined. The problem of whether balloon angioplasty should be performed or not remains difficult. On one hand, it is necessary to acknowledge angiography's limitations, which often result in an underestimation of the stenosis, while on the other hand, we should keep in mind the dissection risk and a successive and necessary transition to PCI with double-stenting, which increases the risk of SB restenosis in the future [7, 10,12]. FFR and iFR methods used nowadays to optimize the tactics of coronary intervention have a number of limiting factors and restrictions $[11,17]$. The perspective alternative to invasive stress tests is introducing the method of ECG from the coronary sinus in order to determine the optimal volume of coronary intervention in the area of CA bifurcation [13, 14, and 15]. In the area of ADA blood supply, reliable dynamics of ST-segment during intervention were registered in the CS 12 lead and in the CS 3-4 lead of the endocardial electrode. During the blocking of blood flow, the minimal ST-segment shifting according to the module was $0.09 \mathrm{mV}$, and the maximal shifting $0.2 \mathrm{mV}$. We did not find a difference in amplitude of the ST-segment in patients with pre-dilatation of stenosis and in those patients who had direct coronarystenting.

The results we obtained during this study completely correspond to the results of the trial held by Yu.L. Shevchenko et al. (2019, 2020), and they confirm the positive results of the use of ECG-CS [13, 14, 15]. The intracardiac CS 3-4 lead bears responsibility for the ischemic dynamics in the area of blood supply of the diagonal branch of the ADA, and corresponds to the topographic characteristics of the coronary sinus and the topic of the myocardial perfusion of the diagonal branches of the ADA [13].

The results of our trial (taking the exactness of ECG-CS relative to ischemia of myocardium in case of comprometation of the side branch of CA into consideration) also correlate with the data we have seen in global medical literature. In his article, Ahn J. et al. (2012) mentioned that in patients having CHD with BL (stenosis of SB before the operation was in $34.8 \pm 17.8 \%$ of cases), $17.8 \%$ of lateral branches had hemodynamically reliable stenosis of BL according to FFR after MV stenting [1]. In the trial conducted by Kang S.J. et al. (2013), Kang noted that after the coronary stenting of the main vessel in patients with BL (SB stenosis before the operation was in $24.8 \pm 16.0 \%$ of cases), $15 \%$ of their lateral branches required additional intervention on the basis of FFR index [9].

In our trial, the use of ECG-CS gave us the possibility to avoid additional intervention in $32(74.4 \%)$ patients who had CHD with MV stenting, having SB protection by a guide wire. In patients who had DB comprometation, the use of the data from intracardiac ECG was helpful to determine the necessary volume of coronary intervention: for 9 patients $(20.9 \%)$ we performed provisional T-stenting; in 2 (4.6\%) patients we found indicators to use a double-stenting method ofPCI.

The ECG-CS method has an exactness relative to myocardial ischemia with invasive loading tests in the case of bifurcational interventions related to pseudo-bifurcation lesions of CA [1,9]. Additionally, the ECSCS method does not have limitations that are typical to FFR and iFR [13].

Therefore, we can say that this brand new approach to determining stenting tactics of bifurcational lesions of CA using ECG-CS is effective.

\section{Limitations related to the investigation.}

There are no limitations related to the investigation. 


\section{Recommendations.}

- It is recommended to use the ECG-CS method in the case of coronary intervention in the area of CA bifurcation if you have the necessary equipment with the described modes.

- The diagnostic criteria of ischemia in the area of blood supply of the ADA diagonal branch is the depression or elevation of the ST segment more than $0.1 \mathrm{mV}$ in the intracardiac CS 3-4 lead of a standard 10-canal electrode.

\section{Conclusion.}

- Electrocardiography from the coronary sinus is a new and efficient method of optimizing tactics of percutaneous coronary intervention in the area of bifurcation of coronaryarteries.

- $\quad$ ECG-CS provides the possibility to determine the degree of SB comprometation in the area of CA bifurcation using a singlestent approach to PCI in patients with stable CHD who have relative indicators.

- The use of the ECG-CS method provides the possibility to discover patients with coronary atherosclerosis when the area of CA bifurcation has a lesion and when it is not possible to avoid the transition to a double-stenting PCImethod.

Conflict of interests: The authors have confirmed the absence of relations that could be interpreted as conflicts of interest.

Financing: This investigation has not received any grant from the governmental, commercial or non-commercial sector.

\section{Abbreviations.}

ADA: Anterior descending artery

BL: Bifurcation lesion

CA: Coronary artery

CHD: Coronary heart disease

DB: Diagonal branch

ECG: Electrocardiography

ECG-CS: Electrocardiography from the coronary sinus

EE: Endocardial electrode

FFR: Fractional flow reserve

iFR: Instantaneous wave-free ratio

MV: Main vessel

MyI: Myocardial ischemia

PBA: Percutaneous balloon angioplasty

PCI: Percutaneous coronary intervention

RAS: Russian Academy of Sciences

SB: Side branch

\section{References}

1. Ahn, J.M. Lee J.Y., Kang S.J., et al. (2012). Functional Assessment of Jailed Dise Branches in Coronary Bifurcation Lesions Using Fractional Flow Reserve. JACC: Cardiovascular Interventions 5 (2): 155-161.
Aidu E.A., Trunov V.G., Titomir L.I., et al. (2006). Electrocardiographic ST segment changes as an indicator for localization of injury potentials. A computer simulation study. Kardiologia 15 (1): 21-24.

3. Aras K., Burton B., Swenson D., et al. (2016). Spatial organization of acute myocardial ischemia. J Electrocardiology 49: 323-336

4. Barth T.J., Griebel M., Keyes D.E., et al. (2006). Computing the electrical activity in the heart. Berlin, Heidelberg: SpringerVerlag. 307.

5. Burton B.M., Aras K.K., Good W.W., et al. (2018). Experimentally derived ischemic zone sourcerepresentations. J. Electrocardiology 51 (4). 725-733.

6. Fallahi, A., Khorram H. G., Kokabi A. (2019). Electrocardiogram signal generation using electrical model of cardiac cell: application in cardiac ischemia. J Med Eng \& Tech 43 (4). 1-10.

7. Gao X. F., Zhang Y. J., Tian N. L., et al. (2014). Stenting strategy for coronary artery bifurcation with drug-eluting stents: a meta-analysis of nine randomised trials and systematic review. EuroIntervention 10 (5). 561-569.

8. Johnston B. M., Coveney S., Chang E. T. Y., et al. (2018). Quantifying the effect of uncertainty in input parameters in a simplified bidomain model of partial thickness ischemia. Med Biol Eng and Computing 56:761-780.

9. Kang, S.J., Kim W.J., Lee J.Y., et al. (2013). Hemodynamic impact of changes in bifurcation geometry after single-stent cross-over technique assessed by intravascular ultrasound and fractional flow reserve. Catheter Cardiovasc Interv 82 (7): 1075-1082.

10. Mallidi J, Lotfi A. Fractional Flow Reserve for the Evaluation of Tandem and Bifurcation Lesions, Left Main, and Acute Coronary Syndromes. Interv Cardiol Clin 4 (4) (2015): 471480.

11. Neumann, F.J., Sousa-Uva M., Ahlsson A., et al. (2019). 2018 ESC/EACTS Guidelines on myocardial revascularization. Eur Heart J. 40 (2): 87-165.

12 Niemela M., Kervinen K., Erglis A., et al. (2011).Randomized comparison of final kissing balloon dilatation versus no final kissing balloon dilatation in patients with coronary bifurcation lesions treated with main vessel stenting: the Nordic-Baltic Bifurcation Study III. Circulation. 123 (1): 79-86.

13. Shevchenko Y.L., Ermakov D.Y. (2020). A New Invasive Method of Continuously Monitoring Myocardial Ischemia in Interventional Cardiology - Electrocardiography from the Coronary Sinus. Cardiology and Cardiovascular Medicine 4: 191-202.

14. Shevchenko Yu.L., Marchak D.I., and Gerashchenko A.V., et al. (2019): Pre-operative preparation and electrophysiological monitoring of the myocardium state with heart endovascular interventions. International Conference Scientific research of the SCO countries: synergy and integration. Participants' reports in English. Part 1, 159-167.

15. Shevchenko Yu.L., Sveshnikov A.V., Marchak D.I., et al. (2019). Electrocardiography of the coronary sinus in intracardiac interventions (in Russian). Bull Pir Nat Med \&Surg Cent 14 (1): 4-11.

16. Shlofmitz E., Jeremias A.FFR in 2017: Current Status in PCI Management. acc.org: ACC Homepage (2017).

17. Soares A., Brown D.L. (2019). The fallacies of fractional flow reserve. International Journal of Cardiology 302:34-35. 